\section{Ignorer bête}

Ignorer stupidement, bêtement

$\lambda$ ignorer haut

\section{Ignorer court}

ignorer haut et court : ignorer totalement

$\lambda$ ignorer haut

\section{Ignorer haut}

Ignorer manifestement (un fait)

Emploi absolu

1938 Si c'est bien là, messieurs, le rôle d'ingénu à quoi le comité me destine, je suis aussitôt à mon aise, et je sais ce que je viens faire : je viens ignorer tout haut (Paul Valéry, Variété IV)

\section{Corpus Web :}

Si les gens préfèrent ne pas se prendre la tête et ne plus RP avec moi, soit. Mais je n'ai encore jamais eu personne me déclarer clairement qu'il déciderait de m'ignorer haut et court [http:// nidhogg.forumactif.org/t70p15-concernant-laguilde-cirel] (30.5.2015)

on roulais pépère à $160 \mathrm{~km} / \mathrm{h}$ sur les autoroutes, à 90 en ville, en ignorant haut et bête les panneaux de signalisation, et en klaxonnant les faux derch qui ce la jouaient à respecter la règlementation... [http://forum.sadur.org/viewtopic. php?t=4587] (30.5.2015)

REMARQUeS : Ignorer haut désigne le fait de ne pas (re)connaitre quelque chose, ou ne pas considérer quelqu'un/quelque chose ouvertement, voire de bouder ouvertement quelqu'un. Le CW illustre l'emploi transitif du verbe. Notons les collocations haut et court et haut et bête. La collocation usuelle haut et court est emphatique, court n'ayant pas un sens clairement identifiable. Haut reste invariable et est modifié par tout.

\section{Imaginer fort}

Imaginer fortement

Transitif

+1365 Et je imagine bien fort

Le gent corps et le bel deport

(Jean Froissart, Poésies [3e tiers XIV]])

\section{CORPuS Web :}

Bon, forcément, l'explosion en sanglots c'est tout de suite moins bien qu'en images, mais faut se l'imaginer fort fort fort hein, pasque moi, Lapin je l'aime ('fin l'ours verte surtout...) et ça fait longtemps qu'il me fait aller du rire aux larmes [http://www.lapin.org/index. php?comms=yes\&number=1] (30.5.2015)

C'est comme dans le film du capitaine crochet faut $s$ imaginer fort fort fort la bouffe pis elle apparais !! [https://www.youtube.com/ watch? $v=$ LKnWS-ivlWw\&index=31\&list=PLC2E9 7832BEF9F49C] (30.5.2015)

L'important est de s'imaginer fort face à une maladie faible. Il arrive aussi que certaines personnes aient des visualisations involontaires et spontanées les aidant parfois à découvrir la cause ou le remède au mal dont ils souffrent [http:// www.santenaturellement.com/Visualisation. $\mathrm{htm}]$ (30.5.2015)

Le clapotis plastique de mes doigts sur ce putain de clavier me remonte à l'âme comme les godillots martelant les flaques sur les chemins de ronde... Ma forteresse s'épuise à s'imaginer forte, tandis que le désordre m'envahit par cascades fumantes... [http://www.atramenta.net/lire/ oeuvre37522-chapitre236954.html] (30.5.2015)

REMARQues : Imaginer fort désigne le fait de se représenter quelque chose en pensées avec force, avec beaucoup de détails et de précision. Fort reste invariable dans son emploi adverbial (v. le premier exemple du CW). Mais quand il est pris au sens de 'quelqu'un s'imagine être fort', il devient un prédicat second qui tend à l'accord (deux derniers exemples du CW). Notons la réduplication fort fort et l'emploi pronominal du verbe dans le CW. Fort est modifié par bien.

\section{Importer tout rond}

Faire venir sans hésiter

Transitif

1972 Gobineau a tout simplement servi de bouc émissaire... Malgré ces deux excellents ouvrages, le poids n'y était pas. Alors on a importé tout rond un écrivain français, Hervé Bazin, le neveu de l'illustre René Bazin (Le Maclean)

REMARQUES : Importer tout rond figure dans un magazine franco-canadien où il renvoie au fait de faire venir quelqu'un de France par une action immédiate, sans autre forme de procès 
('directement, sans plus'). Il s'agit d'une combinaison libre du modifieur lexicalisé tout rond qui connaît plusieurs acceptions contextuelles : 'entièrement' (avaler tout rond), 'directement / sans plus' (comme dans l'exemple), 'sans se faire des soucis / sans gêne’ (dire / voler tout rond).

\section{Imprimer fin}

Imprimer en petites lettres, en petits caractères Transitif

190816 mai 1908.

Les gouvernantes qui lisent, sur un banc, des livres imprimés toujours très fin. Elles s’abîment les yeux (Jules Renard, Journal)

1911 Et les pilules coûtent cher, dans des boîtes avec des cachets, et dedans, sur papier pelure, des prospectus imprimés fin, où il $\mathrm{y}$ a des lettres de malades guéris (Charles-Ferdinand Ramuz, Aimé Pache, peintre vaudois)

1957 un livre de 500 pages, imprimé très fin (Exemple entendu, 27 novembre 1957 / Grundt : 407)

CORPUS Web :

Un post-it blanc sur la partie à aligner, il suffit de projeter l'image de la cible sur le postit. En imprimant fin et en ajustant la netteté, tu as une précision très en dessous du mm [http:// barduport.forumactif.org/t1275-pas-tres-marinmais-nautique-quand-meme-qui-peut-m-aiderdans-mon-choix] (30.5.2015)

Si c'est une $0.4 \mathrm{~mm}$ tu imprime trop fin, il faut que tu imprime en 0.3 [http://forums.reprap. org/read.php?110,463113,463567] (30.5.2015)

C'était une petite Bible de poche, imprimée très fin [http://spirite.free.fr/ouvrages/invisible 18.htm] (30.5.2015)

REMARQUES : Généralement sous la forme d'un participe passé, imprimé fin réfère à un écrit (livre, prospectus) imprimé petit, en petits caractères typographiques. Fin reste invariable. Il est modifié par très. Notons l'emploi de coûter cher.

\section{Incarner faux}

Représenter sans naturel, sans réalisme ou vraisemblance

$\pi$ peindre faux

\section{Incendier ferme}

Se disputer, se quereller fermement

Pronominal

1953 Les greluches d’à côté elles s'incendiaient ferme maintenant (Albert Simonin, Touchez pas au grisbi)

REMARQUES : S’incendier ferme désigne le fait de couvrir, d'accabler quelqu'un de reproches. Sous la forme pronominale, il souligne le caractère réciproque de l'action, ferme renforçant la dureté et la vivacité de la dispute. Ferme reste invarié.

\section{Incliner bas}

s'incliner bas : admirer énormément ; marquer, avoir un profond respect (pour quelque chose ou quelqu'un)

Pronominal

1943 Or j'estime que vous avez agi et parlé en prêtre, et la conscience professionnelle est une qualité aujourd'hui trop rare pour que je ne m'incline pas très bas devant elle, n’importe où je la trouve... vous êtes irré-pro-chable, conclut-il d'un ton sans réplique (Georges Bernanos, Monsieur Ouine)

1958 Un élève du Conservatoire, qui répétait avec lui une pièce de $M$. Jeannot intitulée Le Retour à la terre, déclara un soir avec impétuosité : « Il faut s'incliner très bas devant Ibsen ! » Mon père eut un grand rire : « Eh bien, moi, dit-il, je ne m'incline pas! » (Simone de Beauvoir, Mémoires d’une jeune fille rangée)

\section{CORPUS WEB :}

On ne peut que s'incliner bas devant l'équipe de l'entraîneur fédéral du DTTB, Jörg Rosskopf. Sa domination au niveau européen semble devenir de plus en plus grande [http://fr.butterfly. tt/info/c-e-schwechat-2013/1] (31.5.2015)

Comme tu le sais, je n'ai jamais douté de ton talent mais de là à trouver une correspondance homonymique preque parfaite pour réussir ce défi ultramagnetiquien... Je n'ai plus qu'à m'incliner bas, très bas... [http://www.ultramagnetique. com/les-fleurs-du-dub] (31.5.2015)

Ses cheveux cadrant son visage, elle s'avanca près du couple royale. Elle s'inclina bas [http://orcanie.forumactif.com/t12-festivite-en-1honneur-de-dame-morgause] (31.5.2015) 
REMARQues : Suivi de la préposition devant, s'incliner bas se dit du fait de se courber profondément face à quelqu'un, et, par extension, lui donner des marques de profond respect, d'humilité, reconnaître sa supériorité. Bas reste invariable et est modifié par très. Notons la réduplication progressive bas, très bas.

\section{Incliner profond}

Saluer par une profonde inclinaison

Transitif

1230 Après le fist li enfes de riches dras parer ; Lors n'ot il plus bel home en la crestienté. Atant es Huidelon et son riche barné Qui descendent à terre des destriers sejornés.

Où que il voit Guion, parfont l'a encliné, En son sarasinois mult biau l'a salué

(Gui de Bourgogne, p. 84)

+1250 Et Brun preigne garde des mes,

A la table les face pres.

« Ysengrin, pensez de taillier

Et de la coupe apareillier

Devant vo dame la roïne.

Ysengrin parfont l'en encline » (Le Roman de Renart [2 $2^{\mathrm{e}}$ moitié XIII ${ }^{\mathrm{e}}$, XXIII, 1480)

\section{Corpus Web :}

L'homonyme d'un mot d'une phrase écrite ou même prononcée dépend souvent du récepteur, je devais être fatigué _Duck. Je m'incline profond jusqu'aux mules [http://forum. doctissimo.fr/doctissimo/recits-erotiques/ hetero-termine-sujet_13840_3.htm] (31.5.2015)

Les Cocteau Twins... Voilà un groupe que j’ai raté en concert! $:$ Toutes les pseudo-chanteuses à voix du métal gothique s'inclinent profond devant la voix de Liz Frazer (surtout sur Treasure !) ! [http://www.xsilence.net/forum131129858110.htm?w=radio] (31.5.2015)

La dernière activité enregistrée dans la région fut un tremblement de terre en juillet 1840 dont l'épicentre se situait aux alentours de la gorge d'Ahora, un gouffre incliné profond de 1 $825 \mathrm{~m}$ par rapport au sommet [http://armeniewith-me.skyrock.com/737980247-Mont-Ararat. html] (31.5.2015)

La grotte a une structure en forte pente inclinée profonde d'environ 120m [http://www. info-tourisme-madagascar.com/category/nosregions/sud] (31.5.2015)

REMARQUES : Incliner profond se dit du fait de se courber profondément face à quelqu'un, dans un geste de salutation et/ou de respect. Notons l'emploi pronominal du verbe, plus moderne, dans le CW. Les deux derniers exemples du CW désignent une pente ou un abîme profonds. Profond reste invariable dans la majorité des cas (v. le deuxième exemple du CW), mais dans le dernier exemple du CW il s'accorde avec l'objet au féminin. Notons l'emploi de saluer beau.

\section{Incliner soef}

Incliner doucement, délicatement

Pronominal

+1233 Or ne doit nuns Flori blamer

S'il quiert de son mal medecine.

Souef vers la bele s'encline,

Doucement l'estraint a .II. bras,

En mi la bouche par solas

La baise .VII. fois par loisir (Robert de Blois, Floris et Lyriopé [2 $2^{\mathrm{e}}$ tiers XIII'] ${ }^{\mathrm{e}}$, 964)

REMARQUES : S'incliner soef désignait le fait de se pencher délicatement, lentement vers quelqu'un pour le saluer, lui montrer du respect.

\section{Informer (enformer) dur \\ Donner beaucoup d'informations \\ $\lambda$ enformer dur}

\section{Interroger fort}

(S')interroger beaucoup ; (s')interroger

ouvertement

$\lambda$ interroger haut

\section{Interroger haut}

S'interroger délibérément (sur quelque chose)

Pronominal

1890 Il eut un léger battement de paupières, envahi d'une angoisse sous ce regard qui fouillait son crâne. Sa conscience s'interrogea tout haut (Émile Zola, La Bête humaine)

CoRpus Web :

Emanant d'un petit peuple particulièrement disert, dont la créativité est inversement proportionnelle au nombre, le FTA est un temps où le 
Québec prend sur lui de se parler à lui-même, de s'interroger haut et fort, de s'observer, se rêver, se malmener, s'invectiver et s'oublier - pour mieux se retrouver probablement, se réinventer autrement et ailleurs [http://www.mouvement. net/critiques/critiques/trois-etats-du-quebec] (31.5.2015)

A la veille du 14 juillet, et en plein bras de fer avec Nicolas Sarkozy sur le budget 1994, le ministre de la Défense s'interroge haut et fort sur le bien-fondé de la décision de poursuivre le moratoire sur les essais nucléaires [http://www. lesechos.fr/13/07/1993/LesEchos/16431-034-ECH_ francois-leotard-n-exclut-pas-une-reprise-desessais-nucleaires.htm] (31.5.2015)

Elle quitte la pièce en claquant la porte, laissant une Mylène abasourdie. Celle-ci s'interroge haut et fort : " Mais... Mais qu'est-ce qu'elle a ? » [http://tomtomsblog.canalblog.com/ archives/2012/12/04/25737133.html] (31.5.2015)

REMARQUES : S'interroger haut désigne le fait de se poser à soi-même une ou plusieurs questions de manière consciente et concrète. Notons la collocation haut et fort qui est systématique dans le CW. Haut reste invariable (v. le dernier exemple du CW) et est modifié par tout.

\section{Interrompre net}

Interrompre brutalement, abruptement

Emploi absolu

1843 - Hier j'étais au bal chez Mme la vicomtesse de Beauséant, une cousine à moi, qui possède une maison magnifique, des appartements habillés de soie, enfin qui nous a donné une fête superbe, où je me suis amusé comme un roi...

- Telet, dit Vautrin en interrompant net (Honoré de Balzac, Le Père Goriot)

Transitif

1845 - Diable ! diable !... Écoutez, madame, je vous vois venir..

- Non, monsieur, dit la mère d'Oscar en interrompant net le vieillard, qui par égard pour une belle dame retint le mouvement d'humeur qu'on éprouve à se voir interrompu

(Honoré de Balzac, Un début dans la vie)
1905 Il l'interrompit net:

- Pardon, dit-il, avec une sécheresse glaciale, il faut que je vous quitte

(Romain Rolland, Jean-Christophe. L'Adolescent)

1933 Les petites jambes, dressées vers le plafond, s'incurvaient vers la tête. Les petons retrouvaient les menottes pour une exploration passionnante qu'interrompit net la vue d'un crayon rouge sortant de la poche de l'oncle (Joseph Malègue, Augustin ou Le Maître est là)

1973 Ils ont net interrompu leur engueulade pour me regarder d'un air agacé. Si je les agaçais, ils n'avaient qu'à pas me faire venir, voilà mon sentiment (Jean-Patrick Manchette, Morgue pleine)

1993 J'ai posé la plume, interrompu net mon texte du jour, au thème prémonitoire : message de félicitations au Conseil supérieur du notariat français pour le ving tième anniversaire du fichier central des dernières volontés (Éric Orsenna, Grand Amour)

Pronominal

1884 Lazare qui la soulève, pour qu'elle ne mouille pas ses bottines. Il n'en a pas gros dans les bras, allez ! C'est vrai qu'il y a des hommes qui aiment les os... Véronique s'interrompait net, en sentant près d'elle le tressaillement de Pauline. Sans cesse elle revenait à ce sujet, avec la démangeaison d'en dire davantage (Émile Zola, La Joie de vivre)

1928 L’opérateur, les yeux toujours fixés, j'ignore pourquoi, sur la pendule, lance des appels.

- Il a entendu?

- Non. Mais il parle à Casablanca, on va savoir.

Nous captons en fraude des secrets d'ange. Le crayon hésite, s’abat, cloue une lettre, puis deux, puis dix avec rapidité. Des mots se forment, semblent éclore.

« Note pour Casablanca... » 
Salaud ! Ténériffe nous brouille Agadir ! Sa voix énorme remplit les écouteurs. Elle s'interrompt net.

«... terri six heures trente. Reparti à...» Ténériffe l'intrus nous bouscule encore (Antoine de Saint-Exupéry, Courrier Sud)

1958 Bullit s'interrompit net, et comme pour se punir d'une faute qui m'échappait, mordit brutalement sa lèvre inférieure (Joseph Kessel, Le Lion)

\section{CORpus Web :}

Ainsi en cas de toux sèche (il vaut mieux ne pas interrompre une toux grasse, qui sert à désengorger les bronches), il suffit d'avaler sa salive pour interrompre net la poussée de toux [http:// www.remede-de-grand-mere.fr/2013/01/stoppernet-la-toux] (31.5.2015)

Surgie des coulisses en costume de mousquetaire, Bartoli fait aussitôt délirer la salle, déjà acquise à sa cause : Brava !, Cecilia !, Viva ! fusent de toute part, obligeant l'orchestre à interrompre net l'introduction instrumentale du premier air [http://www.concertonet.com/scripts/review. php?ID_review=9102] (31.5.2015)

Sur les murs de son bureau, dans ses locaux de Void-Vacon dans la Meuse, un cadre façon Wall-street vient rappeler la courbe de croissance de Clair de Lorraine. Une courbe ascendante. Mais qui aurait pu s'interrompre net, en 2008 [http://www.lasemaine.fr/2012/03/01/vincentferry-toujours-un-pas-d-avance] (31.5.2015)

Le terrain a été labouré par des camions de débardage, nous prenons sur la gauche une piste qui part à flanc. Au bout d'un quart d'heure, la piste s'interrompt nette et nous n'avons plus vu de balises rouge et blanche depuis la route... [http:// rando-voyage.over-blog.com/article-hrp-j28-lemas-reste-banyuls-104799288.html] (31.5.2015)

REMARQUES : Interrompre net désigne le fait d'arrêter (quelqu'un) dans son action, dans son activité, de manière brusque et évidente. Dans une conversation, il signifie 'arrêter (quelqu'un) dans son discours, (lui) couper brusquement la parole'. Par extension, il s'applique également dans d'autres domaines comme la santé, la circulation, ou encore la bourse (CW). Net reste invariable dans la majorité des cas, mais il s'accorde avec le sujet dans le dernier exemple du CW. Notons l'antéposition de net dans l'exemple de 1973. Mentionnons l'emploi de en avoir gros dans les bras.

\section{Issir droit}

Sortir juste au moment où quelque chose se passe Intransitif

$\sim 1350$ Moult fuit a grant meschief la duchesse adoubee.

De la citeit issit droit a l'aube crevee ; La ou li joian fuit ait sa voie tornee. Li joians fuit couchiéz droit en une vallee, D'ancoste lui avoit sa haiche et son espee (Lion de Bourges, 1654)

REMARques : On observe déjà très tôt, au XIV ${ }^{e}$ siècle, le déplacement de la modification du verbe, ici : coucher et issir, vers la préposition, ici : droit à/en.

\section{Issir soef}

Sortir lentement, doucement Pronominal

1200 Puis frema bien l'us a la clef De son ostel s'en ist soef (Auberee, 322)

Intransitif

$\sim 1275$ Floriant ist fors de la nef Tout simplement et tot soef, Florete tient par la main nue Qui n'estoit de riens esperdue (Floriant et Florete, 8102)

REMARQUES : Issir soef désignait le fait de quitter un lieu calmement, doucement, précautionneusement. Coordonné avec simplement, soef est modifié par tot. 